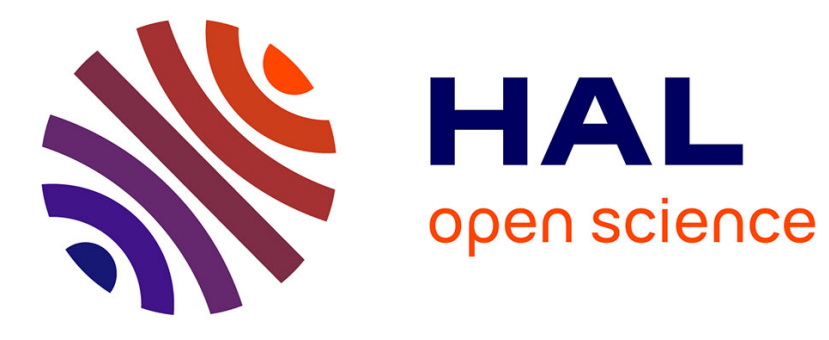

\title{
Tubular Objects Network Detection from 3D Images
}

Nicolas Flasque, Michel Desvignes, Jean-Marc Constans, Marinette Revenu

\section{To cite this version:}

Nicolas Flasque, Michel Desvignes, Jean-Marc Constans, Marinette Revenu. Tubular Objects Network Detection from 3D Images. 4th IEEE Southwest Symposium on Image Analysis and Interpretation, 2000, Austin, Texas, United States. pp.96-100, 10.1109/IAI.2000.839579 . hal-00960750

\section{HAL Id: hal-00960750 https://hal.science/hal-00960750}

Submitted on 18 Nov 2014

HAL is a multi-disciplinary open access archive for the deposit and dissemination of scientific research documents, whether they are published or not. The documents may come from teaching and research institutions in France or abroad, or from public or private research centers.
L'archive ouverte pluridisciplinaire HAL, est destinée au dépôt et à la diffusion de documents scientifiques de niveau recherche, publiés ou non, émanant des établissements d'enseignement et de recherche français ou étrangers, des laboratoires publics ou privés. 


\title{
Tubular Objects Network Detection from 3D Images
}

\author{
N. Flasque $^{(1)}$, M. Desvignes ${ }^{(1)}$, J.M. Constans ${ }^{(2)}$, M. Revenu ${ }^{(1)}$ \\ (1) : Greyc-Image ISMRA, 6 Bd du Maréchal Juin F-14050 CAEN Cedex \\ (2) : Service d'IRM du CHU de Caen, Avenue de la côte de Nacre F-14000 CAEN Cedex \\ e-mail : Nicolas Flasque@sreycismrafr
}

\begin{abstract}
We present an upproach to the tree representation of a tubular objects network. The full-3D tracking algorithm for a single tubular structure is detailed. Detection of bifurcations by a connectivity approach is then exposed.

We show subvoxel accuracy and reliable orientation estimation for the tracking process on synthetic images. Bifurcations are also well detected on a complex synthetic image. Finally, applications of this method on real $3 D$ medical images are shown. The method is particularly suited for processing Magnetic Resonance Angiography of the brain and neck.
\end{abstract}

\section{Introduction}

Spatial resolution of Magnetic Resonance Angiography (MRA) make's it a powerful tool for diagnosis and surgical plannin!. However, image interpretation and visualization l(x)ls are missing, and three-dimensional measurements arc not usually accessible. Flexible visualization of the whole vascular tree and precise quantification of phenomenons like carotid stenosis are applications of in automated processing of MRA [2]. Building an accurate representation of a tubular objects network such is hronchi or blood vessels can provide a substantial holp fir $3 \mathrm{D}$ visualization and quantification.

We prescnt :m approach to the tree representation of a tubular objects nctwork. The main originality of this work is the $3 \mathrm{D}$ crntcrline tracking process which provides subvoxel acruricy and deals with bifurcations. This approach has hin succesfully applied to femoral arteries and cerebral visiulature.

\section{Method}

The tutular wiects network detection is presented in two steps : thi racking process of a single structure and the detection wifurcations which occurs at each step of the trackins privis.

\subsection{Tubular objects tracking}

Tracking is an iterative process with subvoxel accuracy. It detects the centerline of tubular objects using a segmented image. From an original image I, we compute an image $S(\mathrm{I})$. $S(\mathrm{I})$ contains unstructured sets of connected voxels belonging to the objects to be identified.

To insure subvoxel accuracy, tracking has to be processed in a continuous 3D space obtained by interpolation.

From a given point $P_{\mathrm{i}}$ and a given direction $D_{\mathrm{i}}$, a local computation is done in a search area to find the point $P_{\mathrm{i}+1}$ and direction $D_{i+1}$. The search area is a mobile parallelepiped built (Fig. 1) at each step according to $P_{\mathrm{i}}$ and $D_{\mathrm{i}}$ values. The dimensions of the parallelepiped, $L_{\mathrm{i}}, l_{\mathrm{i}}$ and $H_{i}$ are computed dynamically and evolve with estimated object diameter and local curvature.

2.1.1. Detection of point $\mathbf{P}_{\mathbf{i}+\mathbf{1}}$. Let $P_{\mathrm{i}}$ be the current point and $D_{\mathrm{i}}$ the current tracking direction, let $L_{\mathrm{i}}, l_{\mathrm{i}}$ and $H_{\mathrm{i}}$ be the dimensions of the box.

$P_{\mathrm{i}}$ is located at the center of the 'lower' face $\mathrm{F}$ of the parallelepiped (fig. 3).

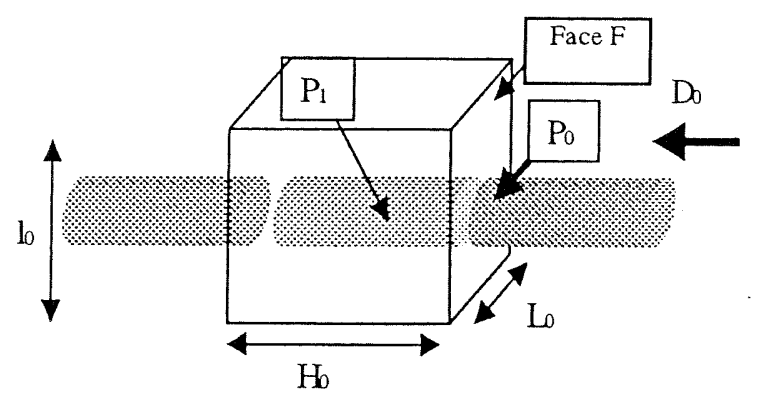

Figure 1 : principle of the mobile parallelepiped. 
Let $V$ be the whole set of binary voxels included in the parallelepiped and $B_{\mathrm{i}}$ the gravity center of those voxels (table 2).

$$
\begin{gathered}
V=\left\{X_{\alpha, \beta, \gamma} / X_{\alpha, \beta, \gamma} \in S(\mathrm{I})\right\} \\
\text { with } \alpha \in\left[-l_{i},+l_{i}\right], \beta \in\left[-L_{i},+L_{i}\right], \gamma \in\left[0,+H_{i}\right] \\
X_{\alpha, \beta, \gamma}=P_{i}+\left(\begin{array}{l}
\alpha \\
\beta \overrightarrow{p_{i}} \\
\gamma
\end{array}\right) \\
B_{i}=\frac{1}{C a r d(V)} \times \frac{\sum_{X \alpha, \beta, \gamma} A_{\alpha, \beta, \gamma} \times X_{\alpha, \beta, \gamma}}{\sum_{X \alpha, \beta, \gamma} A_{\alpha, \beta, \gamma}} \\
A_{\alpha, \beta, \gamma}=1 \text { or } A_{\alpha, \beta, \gamma}=\operatorname{Im}\left(X_{\alpha, \beta, \gamma}\right) \text { or } A_{\alpha, \beta, \gamma}=e^{\operatorname{Im}\left(X_{\alpha, \beta, \gamma}\right)}
\end{gathered}
$$

Table 2: Computation of the set $V$ and of $B_{i}$ point

The point $P_{\mathrm{i}+1}$ and direction $D_{\mathrm{i}+1}$ are given by the following equations (table3):

$$
\begin{aligned}
P_{i+1} & =\frac{\left(k_{p} \cdot P_{i}\right)+B_{i}}{k_{p}+1} \\
\overrightarrow{D_{i+1}} & =\frac{\overrightarrow{D_{i}}+k_{d} \cdot\left(\frac{\overrightarrow{B_{i}-P_{i}}}{\left\|\overrightarrow{B_{i}-P_{i}}\right\|}\right)}{k_{d}+1}
\end{aligned}
$$

Table 3: Computation of $P_{i+1}$ and $D_{i+1}$

Parameters $k_{p}$ and $k_{d}$ are weights between previous and actual values in a prediction-verification paradigm, such as PID controler or a Kalmann filter; $k_{p}, k_{d} \in[0,1]$. These parameters have a fixed value corresponding to the maximal curvature of tubular structures that can be tracked by this technique. For a given set $\left\{k_{p}, k_{d}\right\}$, the maximum curvature that can be estimated by the tracking algorithm is limited by the dimensions of the paralleliped which are computed at each step of the tracking.

2.1.2. Computation of adaptative dimensions. The dimensions $L_{\mathrm{i}}, l_{\mathrm{i}}$ and $H_{\mathrm{i}}$ are calculated at each step of the tracking algorithm to suit the local data with maximal accuracy and speed (evaluated as a discrete derivative between two successive positions). The change of direction between two steps $i$ and $i+1$ is evaluated by the following value :

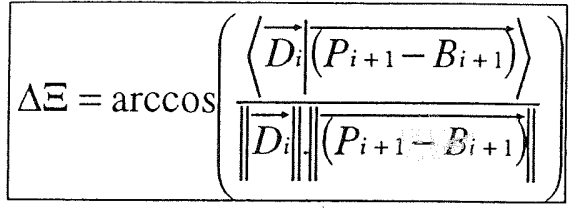

Large values of $\Delta \Xi$ inidicate a significant change of direction between steps $\mathrm{i}$ and $\mathrm{i}+1$. In this case, direction change between $D_{\mathrm{i}}$ and $D_{\mathrm{i}+1}$ must be suited to those abrupt changes and the position update must be as small as possible in order to stay in the correct area. A large number of points must be computed in sinuous areas for a good accuracy.

Furthermore, we must insure that only one tubular structure is tracked at a time by the parallelepiped: dimensions $L_{\mathrm{i}}$ and $l_{\mathrm{i}}$ are computed at each step so that only one connected component is visible through the 'lower' face F. Non circular sections for tubular structures imply that $L_{\mathrm{i}}$ and $l_{\mathrm{i}}$ are computed separately. Dimensions are first reduced, possibly several times, if all the border voxels of the face $F$ have a value of 0 . They are then augmented once if any border pixel of the face $F$ belongs to the main connected component in this face.

$H_{\mathrm{i}}$ is the horizon of the parallelepiped. Straight sections don't require as much sampling points than sinuous ones. $H_{\mathrm{i}}$ evolves inversely proportionnal to the local curvature, estimated by the direction change $\Delta \Xi$ :

$$
H_{i}=k_{h} \cdot e^{-\Delta \Xi}
$$

This dynamic handling of the dimensions insures that the tracking process remains as close as possible to the structure. It also overcomes small disconnections.

\subsection{Detection of bifurcations}

In order to detect bifurcations and end of structures, connnectivity informations are used. Two quantities are computed : NV, the number of volumic connected components inside the parallelepiped, and NS, the number of surfacic connected components at the boundaries of the parallelepiped. Relative values of these quantities indicate whether to check for a bifurcation (Fig. 2b) or not (Fig. 2a). NV and NS are computed at each step of the tracking algorithm.

A single structure with no bifurcation gives values of 1 for NV and 2 for NS. A single structure with a bifurcation will give values of 1 for NV and 3 for NS. Problems arise for values of NV and NS of respectively 2 and 4 : there are at least two possible configurations giving those values, with significant differences in the resulting tree interpretation. In order to avoid misinterpretation of those values, each surfacic component is also labelled with the label of the volumic component it is attached to. We are then able to detect bifurcations with no ambiguity. 


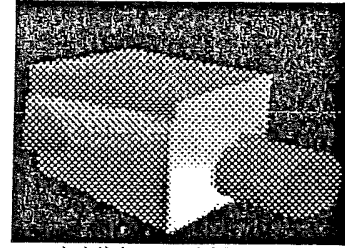

a) $N V=1 ; N S=2$

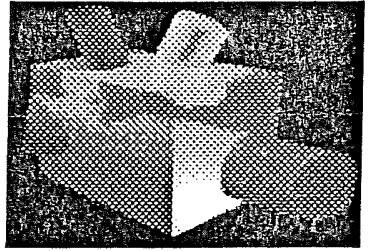

b) $N V=2 ; N S=5$
Figure 2 : Possible voxel configurations inside the parallelepiped with NV and NS values.

End of structures are detected when NS and NV are both equal to 1 . This surface vs. volume connectivity approach theorically allows to detect more than one separation from the structure in a single step (NV $=1$, NS $\geq 4$ ).

\subsection{Tree representation of the network}

Each instance of the tracking step creates a node in the tree. If a bifurcation is detected, a child node is created for each new branch of the bifurcation. A node contains the description of the tubular object by a generalized cylinder. We use two sets of descriptors for the generalized cylinder : a set $\left\{C_{\mathrm{i}}, \mathrm{i} \in[1 . . \mathrm{n}]\right\}$ of center points and a set $\left\{S_{\mathrm{i}}, \mathrm{i} \in[1 . \mathrm{n}]\right\}$ of data on cross-sections. A section $S_{\mathrm{k}}$ corresponds to the point $C_{\mathrm{k}}$.

The $C_{\mathrm{i}}$ points are computed along the second order Bspline interpolating the set $\left\{P_{\mathrm{i}}\right\}$ of detected points. This analytic representation allows resampling and computation of a continuous first derivative $\Delta$ [Far92]. Samples of $\Delta$ at the $C_{\mathrm{i}}$ points $\left(\Delta_{\mathrm{i}}\right)$ give the tangent direction to the $B$-spline curve. The $S_{\mathrm{i}}$ cross-sections are built by interpolation in the plane orthogonal to $\Delta_{i}$ and are designed for quantification and virtual endoscopy. $C_{\mathrm{i}}$ and $\Delta_{i}$ are $3 \mathrm{D}$ vectorial values.

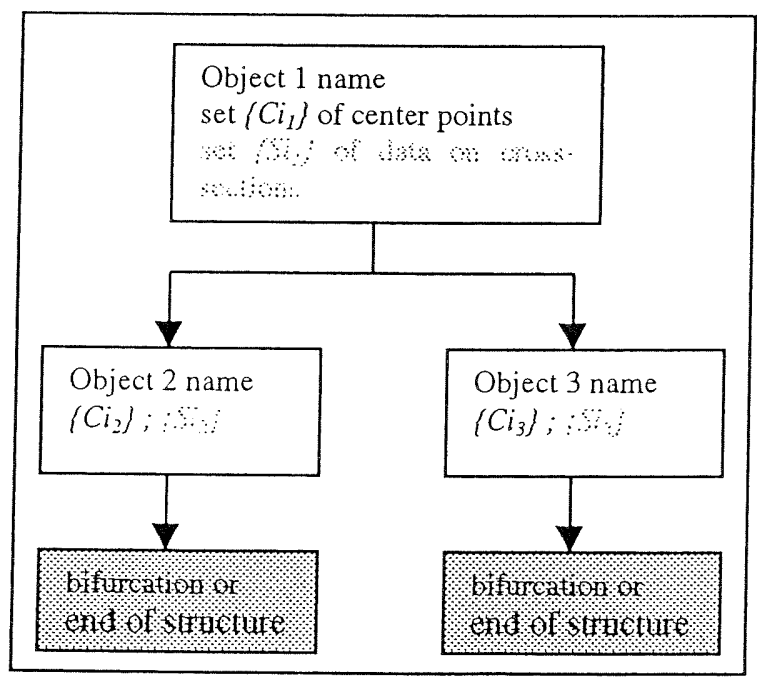

Table 4: Tree representation of a complex network

\section{Results}

Two quantities have to be measured to evaluate the accuracy of the tracking process : the distance between the real and the detected centerline and the orientation difference between those 3D lines.

Images of real 3D structures from various domains don't provide any information about the real centerline position and orientation. We build synthetic images of 3D tubular structures where those values are easily computed. Several structures can be present in a single synthetic image.

Each tubular structure is described by a list of points $\left\{P_{C \mathrm{i}}, \mathrm{i} \in[1 . . \mathrm{m}]\right\}$. The centerline of the structure is the bicubic B-spline curve interpolating the $\left\{P_{C_{i}}\right\}$ set. We compute a set of centerline points $\left\{L_{\mathrm{i}}, \mathrm{i} \in[1 \ldots \mathrm{p}]\right\}$ by resampling the curve, and the set $\left\{\Lambda_{\mathrm{i}}, \mathrm{i} \in[1 . . \mathrm{p}]\right\}$ giving the derivative of the curve for each $L_{\mathrm{i}}$ point.

The distance between the centerlines and the orientation difference are measured between the $\left\{\left(C_{\mathrm{i}}, \Delta_{\mathrm{i}}\right)\right.$, $\mathrm{i} \in[1 . . \mathrm{n}]\}$ sets and $\left\{\left(L_{\mathrm{j}}, \Lambda_{\mathrm{j}}\right), \mathrm{j} \in[1 . . \mathrm{p}]\right\}$ sets by the following formulas :

$$
\begin{gathered}
\Delta P_{i}=\min _{j \in[1 . . p}\left(\| \overrightarrow{C_{i} L_{j} \|}\right) \\
\Delta \Theta i=\arccos \left(\frac{\langle\Delta i \mid \Lambda j\rangle}{\|\Delta i\|\|\Lambda j\|}\right)
\end{gathered}
$$

\subsection{Tracking process}

Three different synthetic image sets have been used to test the precision of the tracking algorithm : a helix shape with constant radii, a helix shape with decreasing main radius (spiral shape) and a Y-fork shape with constant radius (fig. 3). The intensity profile of the generated section is a gaussian.
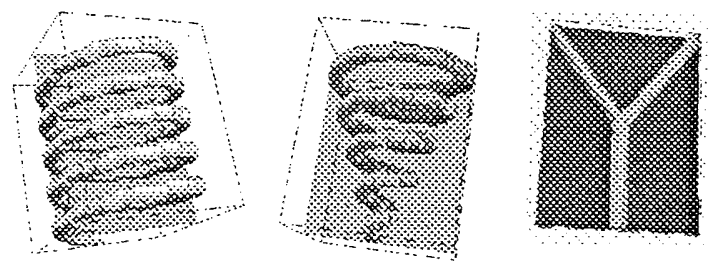

Figure $3: 3 \mathrm{D}$ rendering of the synthetic images used for evaluating the precision of the tracking algorithm

Difference in position between the nearest points $(\Delta P)$ and difference in orientation between those points $(\Delta \Theta)$ are measured between the synthetic object centerline and the detected B-spline curve. Significant results for further quantitative measurements are obtained. 


\begin{tabular}{|c|c|c|}
\hline & $\begin{array}{c}\text { Position error } \\
\Delta P(\mathrm{~mm})\end{array}$ & $\begin{array}{c}\text { orientation error } \\
\Delta \Theta(\text { degree })\end{array}$ \\
\hline $\min$ & 0.19 & 0.05 \\
\hline max & 0.51 & 11.05 \\
\hline mean & $\mathbf{0 . 3 8}$ & $\mathbf{2 . 4 6}$ \\
\hline
\end{tabular}

a) Y-fork shape

\begin{tabular}{|c|c|c|}
\hline & $\begin{array}{c}\text { position error } \\
\Delta P(\mathrm{~mm})\end{array}$ & $\begin{array}{c}\text { orientation error } \\
\Delta \Theta(\text { degree })\end{array}$ \\
\hline $\min$ & 0.01 & 0.10 \\
\hline $\max$ & 0.60 & 11.19 \\
\hline mean & $\mathbf{0 . 2 1}$ & $\mathbf{5 . 5 0}$ \\
\hline
\end{tabular}

b) helix shape

\begin{tabular}{|c|c|c|}
\hline & $\begin{array}{c}\text { position error } \\
\Delta P(\mathrm{~mm})\end{array}$ & $\begin{array}{c}\text { orientation error } \\
\Delta \Theta(\text { degree })\end{array}$ \\
\hline $\min$ & 0.01 & 0.01 \\
\hline $\max$ & 0.35 & 11.25 \\
\hline mean & $\mathbf{0 . 1 9}$ & $\mathbf{4 . 5 7}$ \\
\hline
\end{tabular}

c) spiral shape

Table 5 : Measurements of $\Delta P$ and $\Delta \Theta$ on synthetic images

We estimate the expected error on cross-sectional area measurement as err $=1-\cos ^{2}(\Delta \Theta)$, err is always inferior to $1 \%$. Those measurements show a subvoxel accuracy of 0.2 voxel on a single tubular object centerline tracking, and of 0.4 voxel when a bifurcation is present. This accuracy allows reliable second-order measurements such as diameter and cross-sectionnal area.

\subsection{Detection of bifurcations}

A synthetic image with several connected tubular structures is used to validate the connectivity approach. The angles between the structures vary from $20^{\circ}$ to $70^{\circ}$. All the structures are correctly separated.

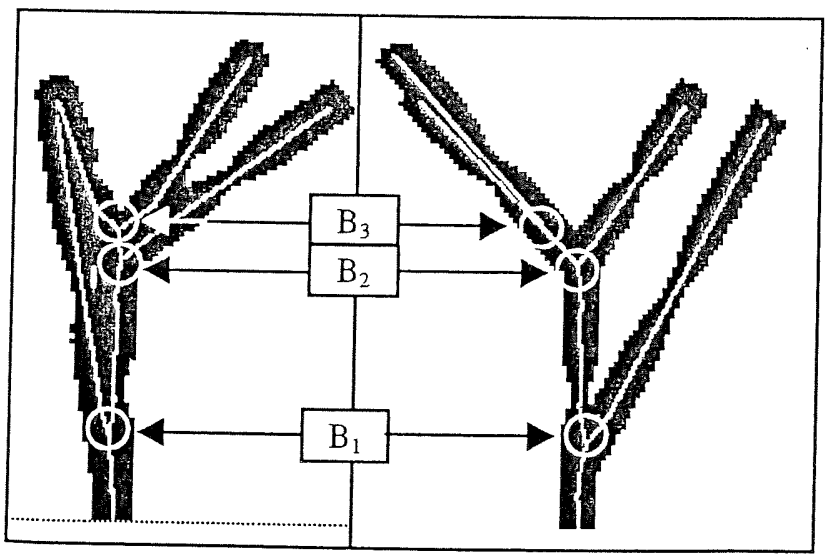

a) left projection

b) front projection

Fig 4: Complete tracking of a tree structure from the bottom on a synthetic image with the detected bifurcations.
The image displayed in fig. 4 is composed of five connected cylindrical structures : a main trunk with a first lone branch (bifurcation $\mathrm{B}_{1}$ ) and an intersection of three other branches at the top (bifurcations $B_{2}$ and $B_{3}$ ). There are seven objects and three bifurcations detected. The top three structures overlap each other, and the original intersection structure of the centerlines becomes a set of two bifurcations. Actually, detection of more than one separation in a single step never occured.

The tubular objects are shrinked in the middle to validate the diameter and cross-sectionnal area measurements currently under progress.

\section{Application}

The cerebral vasculature can be modelled by a network of 3D tubular structures corresponding to the vessels. The tree structure is suited to describe the arterial system. Our work is focused on the internal carotid arteries (left and right). Those vessels are up to 5 voxels large on highresolution images, and their course through the brain is relatively stable. Detecting the carotidian siphon is an important issue for clinicians, as well as providing reliable measures for the cross-sectionnal area.

Furthemore, left and right carotid artery and the vertebral artery interconnect themselves through little vessels in the polygon of Willis. This connection ensures that the arterial blood supply of a particular territory does not depend solely on one artery.

\subsection{Image Acquisition}

Among the numerous imaging techniques available for MRA, we choose a 3D-Time Of Flight, also called inflow-based enhancement, imaging sequence. The main advantages of this imaging technique are a good depiction of the arterial system, the high signal-to-noise ratio and the high resolution achievable [1]. We achieve an isotropic voxel of $1 \mathrm{~mm}^{3}$ along the whole head volume $(256 \times 256 \times 152 \mathrm{~mm})$ in 23 minutes. We use the multislab option in order to maximize the inflow enhancement effect.

\subsection{Global segmentation of the vascular structures}

The purpose of this segmentation is to isolate connected sets of voxels belonging to the blood vessels.

Global segmentation implies three processing steps: the first one is the correction of intensity gaps between slices. Image intensity is not homogeneous through the whole volume. This effect is due to the multislab overlay option [3] that generates clearer strips regularly disposed along the acquisition direction. 
Blood vessels appear as homogeneous regions of high intensity. To enhance those regions, we use diffusion adaptative filtering [5][4] in the second step. Resulting filtering applicable for each voxel depends on two values, rather than on the sole grey-level gradient: the grey level of the processed voxel and the value of the local grey level gradient.

The last step is region gathering of voxels likely to belong to a vessel. We use two criterions for this selection : the intensity of a voxel and its neighbourhood configuration. All voxels of intensity greater than a threshold $I_{1}$ are selected, then candidate voxels are chosen depending on the number of neighbours marked during the first phase. Among those candidate voxels, only those with intensity greater than a threshold $I_{2}$ are kept. $I_{1}$ and $I_{2}$ intensity thresholds are computed on the whole filtered image histogram.

\subsection{Results on MR angiographic images}

No reference centerline is available to evaluate the quality of our tracking algorithm. Results have been visually examined. Vascular tree is well detected. Centerlines are well located for carotid arteries, middle cerebral arteries and the vertebral artery.
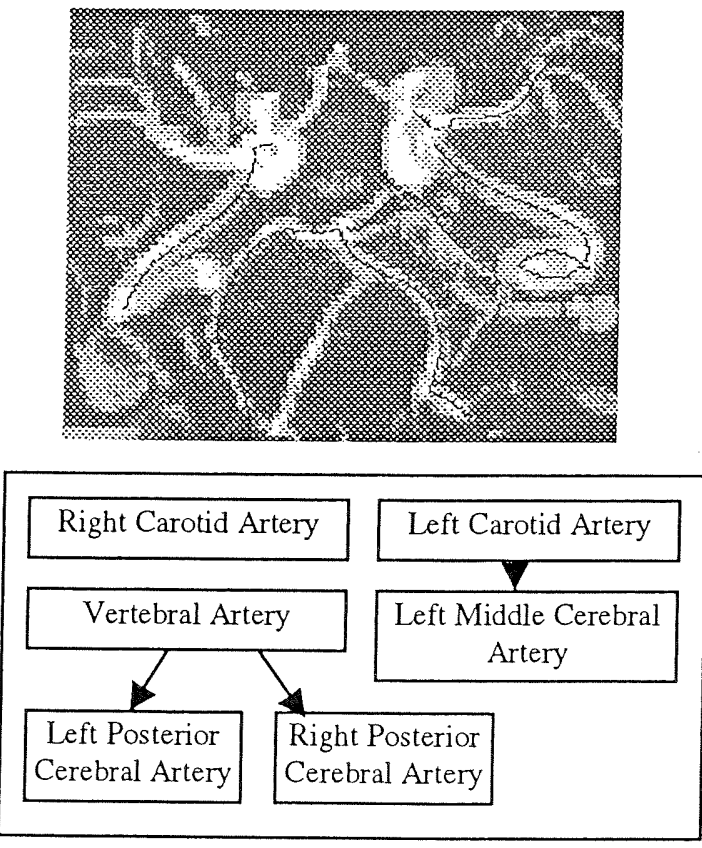

Figure 5 and Table 6: A part of the tracking process on a MRA image of the head and the associated tree representation. Right and left are swapped in the medical nomenclature.

\subsection{Results on Digital Substracted Angiographic (DSA) images}

DSA is another imaging modality for blood vessels. The substraction between two acquisition steps provides an improved contrast on the resulting image.

The image we process is the original image simply thresholded. The image is located at the top of the inferior members (femoral artery).

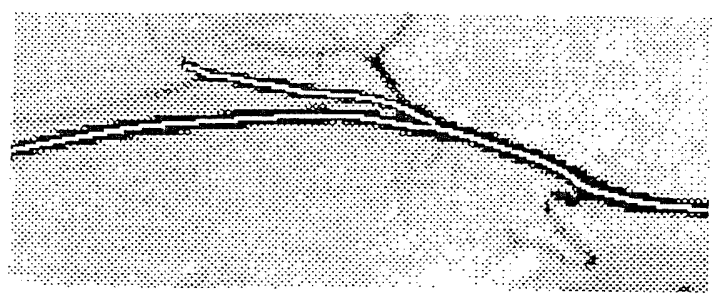

Figure 6: A part of the tracking process on a DSA image of the inferior members.

\section{Conclusion}

In this paper, tracking of tubular objects network from $3 \mathrm{D}$ images has been presented and tested on synthetic images and applied to MRA healthy volunteers images. A $3 \mathrm{D}$ representation of the vascular network is obtained by the detection and the tracking of the vessels centerline. The proposed method is a full $3 \mathrm{D}$ one and handles bifurcations. Its accuracy is better than $0.4 \mathrm{~mm}$ in position and $6^{\circ}$ in orientation on synthetic images.

The tracking algorithm has also been succesfully tested on Digital Substracted Angiograms (DSA) of inferior members area. Current works focus on 2D and 3D quantilication of vessel diameter and cross-sectionnal area to hèlp stenosis detection.

\section{References}

[1]Hougeveen R., Bakker C., Viergever M. (1998), Limits to the accuracy of Vessel Diameter Measurement in MR Angiography, JMRI 8 (6), pp 1228-1239.

[2]Verdonck B. (1996) Blood vessel segmentation, quantification and visualization for $3 D \mathrm{MR}$ and spiral $\mathrm{CT}$ angiography, Ph.D thesis, ENST, Paris.

[3]Korach G., Munier T. and Vignaud J. (1993) Manuel de techniques de l'Imagerie par Résonance Magnétique. Masson, Paris.

[4]Perona P. and Malik J. (1990) Scale-Space and edge detection using anisotropic diffusion, IEEE Transactions on Pattern Analysis and Machine Intelligence, 12(7) pp. 629-639.

[5]Krissian K., Malandain G., Ayache N., Vaillant R. and Trousset Y. (1998) Model based Multiscale Detection of 3D vessels, IEEE Computer Vision and Pattern Recognition, pp 7882. 